\title{
Determination of adequate pelvic lymph node dissection range for Japanese males undergoing radical prostatectomy
}

\author{
NOBUKI FURUBAYASHI ${ }^{1}$, TAKAHITO NEGISHI ${ }^{1}$, HIDENORI IWAI $^{1}$, KEI NAGASE $^{1}$, \\ KENICHI TAGUCHI ${ }^{2}$, MOTOTSUGU SHIMOKAWA ${ }^{3}$ and MOTONOBU NAKAMURA ${ }^{1}$ \\ Departments of ${ }^{1}$ Urology and ${ }^{2}$ Pathology; ${ }^{3}$ Institute for Clinical Research, \\ National Kyushu Cancer Center, Fukuoka 811-1395, Japan
}

Received January 8, 2016; Accepted February 20, 2017

DOI: $10.3892 /$ mco.2017.1204

\begin{abstract}
The present study aimed to determine the adequate pelvic lymph node dissection (PLND) range for Japanese males undergoing radical prostatectomy. A total of 467 Japanese patients who underwent antegrade radical prostatectomy at the National Kyushu Cancer Center (Fukuoka, Japan) were retrospectively reviewed. The patients were divided into two groups according to the PLND extent: The standard (obturator + internal iliac nodes) group and the expanded (standard + additional nodes) group, which accounted for $64.5 \%$ (301/467) and $35.5 \%(166 / 467)$ of the patients, respectively. No differences were observed in the preoperative and postoperative characteristics of the two groups. In addition, there was no difference in PSA recurrence between the two groups. There were no differences between the standard and expanded groups in the low-, intermediate- and high-risk groups $(\mathrm{P}=0.1456$, $\mathrm{P}=0.1581, \mathrm{P}=0.2125$, respectively). The median number of lymph node dissection was 13 and 19, in the standard and expanded groups respectively $(\mathrm{P}<0.0001)$. However, regarding the number of lymph node metastases and the rate of patients with lymph node metastasis, no significant difference was observed between the standard and expanded groups $(\mathrm{P}=0.4219$ and $\mathrm{P}=0.4257$, respectively). According to multivariate analysis, a significant difference in the presence of lymph node metastasis (hazard ratio 3.547; $\mathrm{P}=0.0247$ ), but not in the PLND extent, was detected in patients with prostate specific antigen failure $(\mathrm{P}=0.0655)$. When expanding the dissection extent, the number of dissected lymph nodes increases, but is not associated with the number or rate of positive lymph nodes. Thus, the current dissection range is considered to be appropriate for Japanese men undergoing radical prostatectomy.
\end{abstract}

Correspondence to: Dr Nobuki Furubayashi, Department of Urology, National Kyushu Cancer Center, Notame 3-1-1, Minami-ku, Fukuoka 811-1395, Japan

E-mail: furubayashi.n@nk-cc.go.jp

Key words: prostate specific antigen, prostate specific antigen recurrence, pelvic lymph node dissection, prostate cancer, radical prostatectomy

\section{Introduction}

Pelvic lymph node dissection (PLND) represents the most accurate and reliable staging procedure for the detection of lymph node invasion in prostate cancer (1). For PLND in radical prostatectomy (RP), its adaptation, significance and dissection range have been previously discussed (2). The European Association of Urology (EAU) and National Comprehensive Cancer Network (NCCN) guidelines recommend determining the adaptation of PLND using nomograms to predict the risk of preoperative lymph node metastases when performing PLND, and extended PLND is considered desirable if cases are evaluated as an adaptation of PLND $(1,3)$. However, to date, no consensus has been reached. Therefore, to assess the association between the range of PLND and prostate specific antigen (PSA) failure, a retrospective investigation was conducted using the clinicopathological data of patients who underwent $\mathrm{RP}$, but had not received preoperative treatment.

\section{Materials and methods}

Patient characteristics and risk-group classification. Between August 1998 and May 2013,638 consecutive patients with clinically localized prostate cancer underwent RP at the National Kyushu Cancer Center (Fukuoka, Japan) and were reviewed. $\mathrm{RP}$ was performed in an open retropubic manner in all cases. In total, 171 patients were excluded from this study, including 151 patients due to a past history of receiving hormone therapy, 14 patients due to no PLND being performed and 6 patients due to unclear biopsy or prostatectomy specimen findings. The patients were classified into three risk groups according to the D'Amico criteria (4). Two pathologists evaluated the degree of malignancy in the biopsy and prostatectomy specimens according to the 2005 International Society of Urological Pathology Consensus Conference on Gleason grading system and determined the pathological stage based on the 2009 Tumor-Node-Metastasis classification $(5,6)$.

PLND technique. All patients underwent standard PLND as a minimum, which was performed along the lower edge of the external iliac vein, the caudal limit being the deep circumflex iliac vein and femoral canal, preserving the lymphatics overlying the external iliac artery. The proximal border was bifurcation 
of the common iliac artery, and all tissue in the angle between the external and internal iliac arteries and obturator nerve was removed. All fatty, connective and lymphatic tissue of the obturator fossa along the obturator muscle was removed, leaving the obturator nerve and vessels bare. Subsequently, the internal iliac artery and, as far as possible, the internal iliac vein were skeletonized up to the obturator arteriovenous branch section. The patients were subdivided into two subgroups according to the lymph node dissection technique: Standard PLND and expanded (extended + more extended) PLND. The technique for extended PLND included the technique for standard PLND as well as the lymphatics overlying the external iliac artery and vein, until reaching the genitofemoral nerve laterally. More extended PLND included the technique of extended PLND as well as the lymphatics overlying the common iliac artery up to the ureteric crossing cranially.

Tissue processing and PSA level determination. The RP specimens were fixed in $15 \%$ neutral buffered formalin (Wako Pure Chemical Industries, Ltd., Osaka, Japan) for 48-96 h at room temperature, and whole organ prostate specimens were serially sectioned perpendicular to the rectal surface at $5 \mathrm{~mm}$ intervals. Sections that were predominantly caudal and cephalic were cut in the sagittal plane at $5 \mathrm{~mm}$ intervals in order to assess the bladder neck and apical margins. The specimens were subsequently embedded in paraffin, cut into $5 \mu \mathrm{m}$ sections and stained with hematoxylin and eosin. Extraprostatic extension was defined as the extension of the tumor from the prostate to the periprostatic soft tissue. The presence of tumor cells at the stained resection margin was defined as a positive resection margin. The follow-up schedule after RP involved a PSA assay performed every 3 months for the first 2 years, followed by every 4 months for the next 3 years and every 6 months thereafter. Disease recurrence or PSA failure was determined as the time point when the serum PSA level was $>0.2 \mathrm{ng} / \mathrm{ml}$, and RP was performed if the PSA level did not decrease below $0.2 \mathrm{ng} / \mathrm{ml}$ following surgery. A number of patients who underwent RP were subsequently treated with radiation and/or hormone therapy prior to the serum PSA level exceeding $0.2 \mathrm{ng} / \mathrm{ml}$. Therefore, in these patients, the time point of adjuvant therapy was defined as the date of disease recurrence. All patients provided their written informed consent to participate in this study, and the study protocol was approved by the Ethics Committee of the National Kyushu Cancer Center (Fukuoka, Japan).

Statistical analysis. Statistical analyses were performed using the $\mathrm{JMP}^{\circledR}$ Pro, version 11.0.0 software package (SAS Institute, Inc., Cary, NC, USA). The PSA failure-free rate was determined according to the Kaplan-Meier method, and the significance of clinicopathological parameters associated with PSA failure was assessed using the Cox proportional hazards regression model. The $\chi^{2}$ test and Mann-Whitney $U$ test was used to assess the differences between standard PLND and expanded PLND. $\mathrm{P}<0.05$ was considered to indicate a statistically significant difference.

\section{Results}

Clinicopathological characteristics. The clinicopathological characteristics according to the PLND technique used are presented in Table I. All patients were Japanese (median age, 66 years; range, 47-77), and the PSA levels ranged between 0.623 and $39.413 \mathrm{ng} / \mathrm{ml}$ (median, $7.486 \mathrm{ng} / \mathrm{ml}$; normal range $<4.0 \mathrm{ng} / \mathrm{ml}$ ). The median follow-up period after surgery was 53.8 months. According to the PLND technique used, the standard PLND group and the expanded group contained 301 $(64.5 \%)$ and $166(35.5 \%)$ patients, respectively. No differences were observed in the preoperative characteristics, including age, preoperative PSA, clinical tumor stage and biopsy Gleason score, between the groups (Table I). There were also no differences in postoperative characteristics, such as pathological tumor stage, final Gleason score, extraprostatic extension, resection margin, seminal vesicle invasion and lymph node metastasis between the groups. In addition, there was no significant difference in PSA recurrence between the two groups. Furthermore, lymph node metastasis was not observed in any patient in the low-risk group, but was detected in 5 patients in the intermediate-risk group and 8 patients in the high-risk group.

Association between the lymph node dissection number and lymph node metastasis for each PLND technique. In total, 301 patients $(64.5 \%)$ underwent standard PLND and 166 patients $(35.5 \%)$ underwent expanded PLND (Table II). The median number of dissected lymph nodes was 13 and 19, respectively, and there was a significant difference between the standard PLND and expanded PLND groups $(\mathrm{P}<0.0001$; Table II). Lymph node metastasis was observed in 7 patients $(2.3 \%)$ in the standard PLND group and 6 patients $(3.6 \%)$ in the expanded PLND group. With regard to the number of lymph node metastases and the rate of lymph node metastasis, no significant difference was observed between the standard PLND and expanded PLND groups $(\mathrm{P}=0.4219$ and $\mathrm{P}=0.4257$, respectively).

PSA failure-free survival rate of each dissection template according to risk group classification. According to the D'Amico criteria, 47 (15.6\%), 157 (52.2\%) and 97 (32.2\%) of the patients in the standard PLND group, and 58 (34.9\%), 56 (33.7) and 52 (31.4) patients in the expanded PLND group were classified into the low-, intermediate- and high-risk groups, respectively. In the low-risk groups, the 5-year PSA failure-free rates in the standard and expanded PLND groups were 100 and $94.4 \%$, respectively (Fig. 1). In the intermediate-risk groups, the 5-year PSA failure-free rates in the standard and expanded PLND groups were 88.1 and $91.1 \%$, respectively (Fig. 1). In the high-risk groups, the 5-year PSA failure-free rates in the standard and expanded PLND groups were 67.6 and $78.9 \%$, respectively (Fig. 1). There was no significant difference in the frequency of patients in the low-, intermediate- and high-risk groups between the standard and expanded PLND categories $(\mathrm{P}=0.1456, \mathrm{P}=0.1581$ and $\mathrm{P}=0.2125$, respectively).

Association between patient characteristics and PSA failure. According to the Cox proportional hazards analysis, all characteristics without a preoperative variable, such as age, were significant predictors based on the univariate analysis (Table III). In the multivariate analysis, significant differences were identified in the preoperative characteristics, including preoperative PSA, biopsy Gleason score and clinical tumor 
Table I. Clinicopathological characteristics of patients.

\section{Extent of PLND}

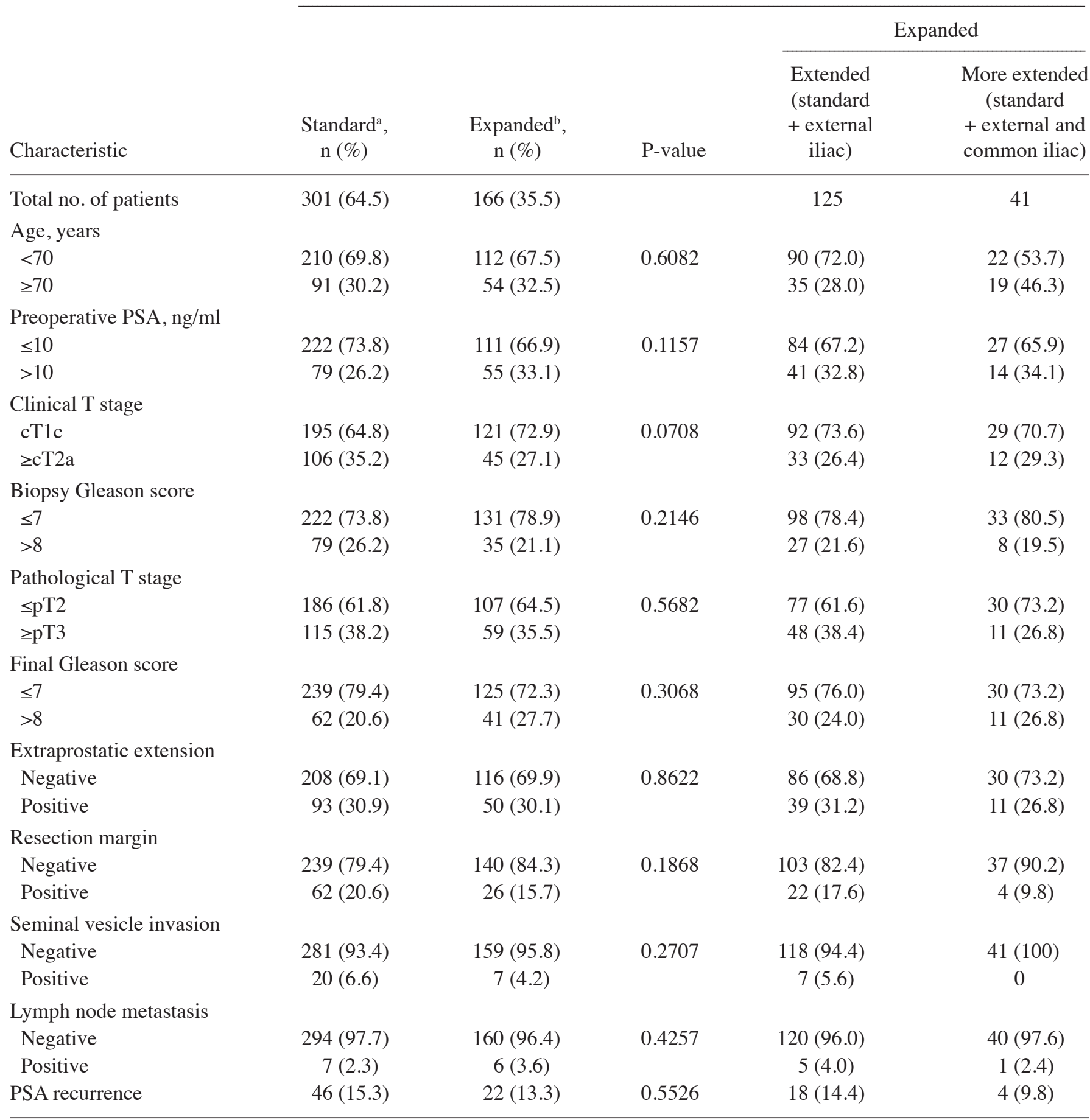

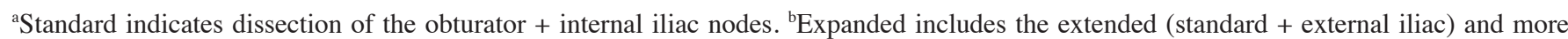
extended (standard + external and common iliac) groups. Clinical and pathological staging was based on the 2009 Tumor-Node-Metastasis classification. P-values calculated using $\chi^{2}$ test. PLND, pelvic lymph node dissection; PSA, prostate-specific antigen.

stage, and in the postoperative characteristics, such as pathological tumor stage, resection margin positive and lymph node metastasis, between the patients with and without PSA failure (Table III). A significant difference in PSA failure between the PLND techniques was only observed in the univariate analysis $(\mathrm{P}=0.0312)$; no significant differences were detected in the multivariate analysis $(\mathrm{P}=0.0655)$.

\section{Discussion}

In patients undergoing RP for prostate cancer, PLND is the most accurate and reliable lymph node staging procedure, as imaging techniques have be demonstrated to be insufficient (7-9). However, to date, no consensus has been reached regarding the extent of PLND required to achieve optimal 
Table II. The association between the lymph node dissection number and lymph node metastasis in the each technique of PLND.

\section{Extent of PLND}

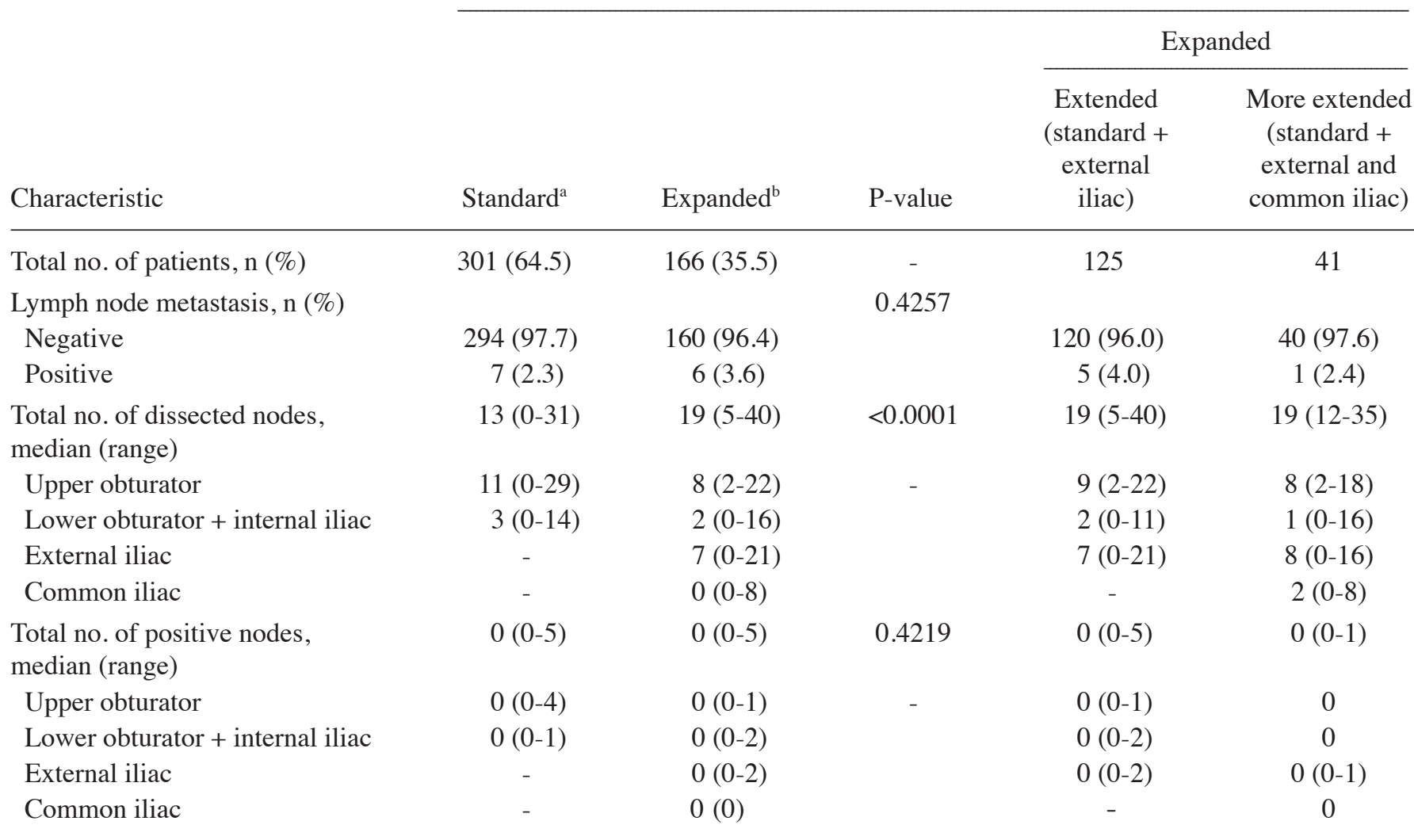

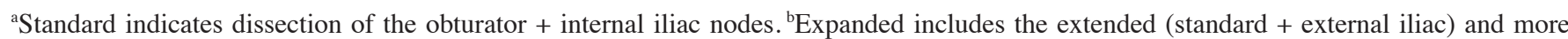
extended (standard + external and common iliac) groups. P-values calculated using $\chi^{2}$ test and Mann Whitney U test. PLND, pelvic lymph node dissection.

lymph node staging. Previous studies have argued that the extent of PLND may be directly correlated with a higher rate of $\mathrm{pN} 1$ stage cases $(10,11)$. Conversely, another study reported no difference in the rate of $\mathrm{pN} 1$ stage cases between patients who received limited and extended PLND (12). Thus, the extent of PLND has varied by era, institution and individual urologist due to the lack of consensus regarding its anatomical extension. In recent years, standard PLND, which is similar (but not identical) to extended PLND in the NCCN guidelines, has been performed at the National Kyushu Cancer Center. When RP was initially performed at this institution, all cases underwent more extended PLND, which includes the common iliac, external, obturator and internal lymph nodes, as there was no consensus among the urologic surgeons with regard to the extent of PLND, and performing PLND with an increased range may allow for the surgical resection of microscopic lymph node metastases. However, even including cases receiving preoperative hormone therapy, the proportion of lymph node metastasis and PSA recurrence rate following RP at the National Kyushu Cancer Center institution were lower compared with previous studies $(13,14)$. Therefore, the range of lymph node dissection was gradually reduced to extended PLND, and finally standard PLND, regardless of the D'Amico risk classification, as more RP procedures were performed. A survival advantage with more extensive lymphadenectomy has been suggested by several studies, potentially due to the elimination of microscopic metastases (15-18). The purpose of the current study was to evaluate the adequate PLND technique for prostate cancer in RP. Compared with a multicenter study, the advantage of this single institutional study is that all of the operations were performed by or under the supervision of urological surgeons who performed standardized surgery, resulting in negligible differences in the dissection of area and methods.

As presented in Table I, the patients were classified into two groups according to the range of PLND: Standard PLND (obturator + internal iliac) and expanded PLND (standard + additional nodes) groups, accounting for 64.5 (301/467) and $35.5 \%$ (166/467) of the patients, respectively. No differences were observed in the preoperative and postoperative characteristics. In addition, there was no difference in PSA recurrence between the two groups. The range of extended PLND differs according to the guidelines used. According to the NCCN guidelines, extended PLND includes the removal of all node-bearing tissue from the area bounded by the external iliac vein anteriority, the pelvic side wall laterally, the bladder wall medially, the floor of the pelvic posteriorly, Cooper's ligament distally and the internal iliac artery proximally (3). On the other hand, according to the EAU guidelines, extended PLND includes the removal of the nodes overlying the external iliac 

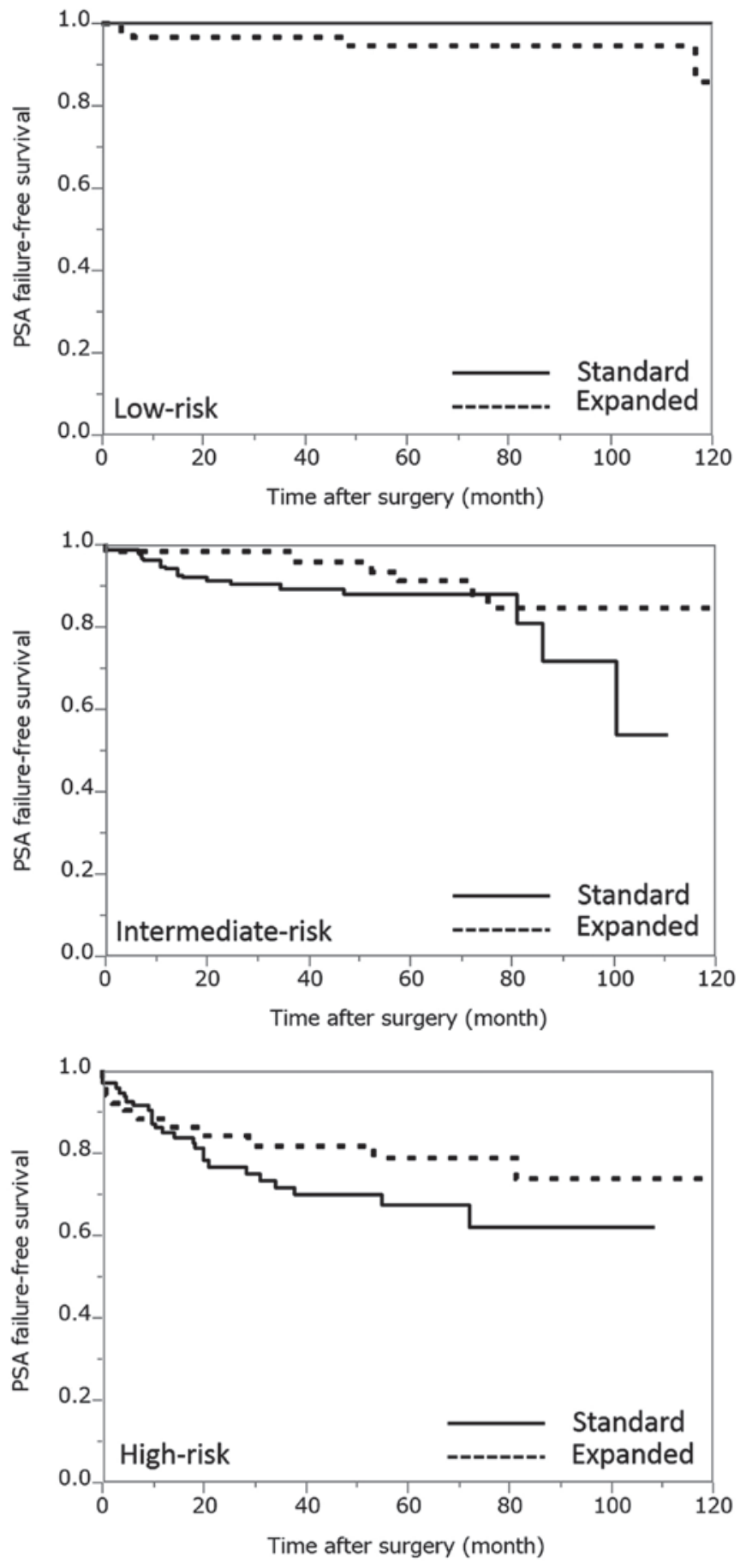

Figure 1. The PSA failure-free survival rate of each dissection technique according to each risk group classification. PSA, prostate specific antigen.

artery and vein, the nodes within the obturator fossa located cranially and caudally to the obturator nerve and the nodes medial and lateral to the internal iliac artery (1). The major difference between these guidelines is the range of extended PLND, that is, whether the nodes overlying the external iliac artery were resected. At the National Kyushu Cancer Center, the standard PLND range is similar (but not identical) to the extended PLND range in the NCCN guidelines, as it was performed along the lower edge of the external iliac vein, so that the nodes overlying the external iliac vein were not resected. The correlation between the lymph node dissection number and lymph node metastasis number was examined for each technique of PLND (Table II). By decreasing the dissected area from expanded PLND to standard PLND, the number of dissected lymph nodes decreased significantly $(\mathrm{P}<0.0001)$. However, with regard to the number of lymph node metastases and the rate of patients with lymph node metastasis, no significant difference was observed between the standard PLND and expanded PLND groups $(\mathrm{P}=0.4219$ and $\mathrm{P}=0.4257$, respectively). A greater number of lymph nodes are able to be dissected when the dissection extent is increased; however, the expansion of the dissection range was not associated with the number or rate of positive lymph nodes detected. According to these results, the present study hypothesizes that there is no need to expand the dissection range further than the standard technique of PLND at the National Kyushu Cancer Center.

The present study subsequently examined the PSA failure-free survival rates of each dissection technique according to each risk group classification (Fig. 1). In the intermediate- and high-risk groups, the 5-year PSA failure-free rates for standard PLND were lower than that of expanded PLND. The risk of lymph node metastasis among the patients in the low-risk group was minimal $(1,19)$. Thus, the therapeutic role of PLND remains unclear and these guidelines indicate that extended PLND may only be recommended for intermediate-, high-risk or highly suspicious patients. However, the results of the present study revealed that there was no significant difference in the outcomes after standard and expanded PLND, not only in the low-risk group but also in the intermediate- and high-risk groups $(\mathrm{P}=0.1456, \mathrm{P}=0.1581, \mathrm{P}=0.2125$, respectively). According to these results, the current study speculates that it is not necessary to expand the dissection range further than the standard technique of PLND at the National Kyushu Cancer Center, even in intermediate- and high-risk patients.

The correlation between patient characteristics and PSA failure were next examined in the RP cases (Table III). First, all factors excluding age were determined to affect PSA recurrence in the univariate analysis. Ultimately, PSA, cT and biopsy Gleason score were factors used in risk classification, and pT, resection margin and lymph node metastasis were factors affecting PSA recurrence in the multivariate analysis. Lymph node metastasis was a significant factor, however, the dissection range was not according to the multivariate analysis $(\mathrm{P}=0.0655)$.

In the current study, it was possible to dissect a greater number of lymph nodes by widening the range of lymph node dissection. However, widening the range of lymph node dissection was not linked to an increase in lymph node metastasis, thus it may not affect PSA failure after RP. According to these results, the National Kyushu Cancer Center's standard dissection range for prostate cancer is considered to be reasonable; however, there is a small difference between this standard dissection range and the extended dissection range around the femoral vein between the NCCN and EAU guidelines.

RP has been applied as one of the local radical therapy modalities for prostate cancer cases which have no metastases, including to the lymph nodes, in preoperative image evaluations. Therefore, it is very rare for surgeons to perform PLND while recognizing hard and swollen lymph nodes, which are suspected of lymph node metastasis during surgery. Namely, lymphadenectomy in prostate cancer occurs when surgeons determine the dissection area according to the anatomical 
Table III. Association between patient characteristics and PSA failure.

\begin{tabular}{|c|c|c|c|}
\hline Characteristics & Hazard ratio & $95 \%$ confidence interval & P-value \\
\hline \multicolumn{4}{|l|}{ Univariate analysis } \\
\hline Age $<70$ vs. $\geq 70$ years & 1.261 & $0.759-2.047$ & 0.3624 \\
\hline Preoperative PSA $\leq 10$ vs. $>10$ & 3.134 & $1.936-5.074$ & $<0.0001$ \\
\hline Clinical T stage, cT1c vs. $\geq \mathrm{T} 2 \mathrm{a}$ & 3.270 & $2.023-5.346$ & $<0.0001$ \\
\hline Biopsy Gleason score $\leq 7$ vs. $>8$ & 3.658 & $2.257-5.916$ & $<0.0001$ \\
\hline Pathological T stage, $\leq$ pT2 vs. $\geq$ pT3 & 5.944 & $3.516-10.519$ & $<0.0001$ \\
\hline Final Gleason score $\leq 7$ vs. $>8$ & 3.621 & $2.242-5.835$ & $<0.0001$ \\
\hline Extraprostatic extension & 3.822 & $2.361-6.277$ & $<0.0001$ \\
\hline Resection margin & 3.159 & $1.908-5.137$ & $<0.0001$ \\
\hline Seminal vesicle invasion & 5.179 & $2.636-9.362$ & $<0.0001$ \\
\hline Lymph node metastasis & 5.792 & $1.995-13.388$ & 0.0029 \\
\hline Range of PLND, standard vs. expanded & 0.563 & $0.323-0.951$ & 0.0312 \\
\hline \multicolumn{4}{|l|}{ Multivariate analysis } \\
\hline Preoperative PSA $\leq 10$ vs. $>10$ & 1.874 & $1.124-3.119$ & 0.0162 \\
\hline Clinical T stage, cT1c vs. $\geq \mathrm{cT} 2 \mathrm{a}$ & 2.780 & $1.686-4.631$ & $<0.0001$ \\
\hline Biopsy Gleason score $\leq 7$ vs. $>8$ & 2.399 & $1.454-3.954$ & 0.0007 \\
\hline Pathological T stage, $\leq$ pT2 vs. $\geq$ pT3 & 3.038 & $1.648-5.766$ & 0.0003 \\
\hline Resection margin & 1.877 & $1.068-3.275$ & 0.0289 \\
\hline Lymph node metastasis & 3.547 & $1.202-8.424$ & 0.0247 \\
\hline
\end{tabular}

Clinical and pathological staging was based on the TNM classification (2009). PSA, prostate specific antigen; PLND, pelvic lymph node dissection.

index, and dissect the connective tissue, including the lymph nodes, as much as possible. Then, the presence or absence of pathologic lymph node metastasis is determined by observing lymph nodes from the excised connective tissue. In some series, the number of nodes removed during lymphadenectomy has been significantly correlated with time to disease progression (13). In one population-based study with a 10-year follow-up, patients undergoing excision of at least 10 nodes (node-negative patients) had a lower risk of prostate cancer-specific mortality at 10 years than those who did not undergo lymphadenectomy (15). However, the number of dissected lymph nodes is considered to be a secondary result, as the number of dissected lymph nodes is also affected by pathological examinations (20), and the secure removal of connective tissue including the lymph nodes in the dissection area is likely to be more important than the number of dissected lymph nodes. An expansion of the pelvic cavity is required by urologists so that larger quantities of connective tissue, including lymph nodes, are able to be removed, and the dissection of connective tissues must be performed to the greatest extent possible, in order to eliminate localized microscopic metastases surgically. Though it was not shown in the table of this article, there were no patients with lymph node metastasis in the low-risk group. In addition, the rates of patients with lymph node metastasis were low (2.3 and 3.6\% in the standard and expanded group, respectively), but this does not indicate whether PLND may surgically eliminate microscopic lymph node metastases that are not able not be pathologically diagnosed as lymph node metastases. Therefore, PLND may be necessary in the RP cases with intermediate- and high-risk, but not for patients with low-risk disease. The NCCN and EAU guidelines also indicate that extended PLND must only be recommended for intermediate- and high-risk patients, consistent with the hypothesis of the present study.

In conclusion, at the National Kyushu Cancer Center, the reduced the range of PLND has been gradually reduced over time, and standard PLND is routinely performed. Although a greater number of lymph nodes are able to be dissected when the dissection extent is greater, the expansion of the dissection range was not associated with the number or rate of positive lymph nodes. Therefore, the current dissection range is considered to be appropriate for Japanese males undergoing radical prostatectomy.

\section{References}

1. European Association of Urology: Guidelines on prostate cancer. http://uroweb.org/guideline/prostate-cancer/. Accessed October 1, 2015.

2. Cookson MS: Should pelvic lymph node dissection be performed with radical prostatectomy? J Urol 183: 1284-1285, 2010.

3. National Comprehensive Cancer Network: Guidelines on prostate cancer. http://www.ncen.org/professionals/physician gls/f guidelines.asp\#prostate/. Accessed October 1, 2015.

4. D'Amico AV, Whittington R, Malkowicz SB, Schultz D, Blank K, Broderick GA, Tomaszewski JE, Renshaw AA, Kaplan I, Beard CJ and Wein A: Biochemical outcome after radical prostatectomy, external beam radiation therapy, or interstitial radiation therapy for clinically localized prostate cancer. JAMA 280: 969-974, 1998.

5. Epstein JI, Allsbrook WC Jr, Amin MB and Egevad LL; ISUP Grading Committee: The 2005 International Society of Urological Pathology (ISUP) consensus conference on gleason grading of prostatic carcinoma. Am J Surg Pathol 29: 1228-1242, 2005. 
6. Sobin LH, Gospodarowicz MK and Wittekind Ch (eds): TNM Classification of Malignant Tumors. 7th edition. Wiley Blackwell, Oxford, 2009.

7. Katz S and Rosen M: MR imaging and MR spectroscopy in prostate cancer management. Radiol Clin North Am 44: 723-734, 2006.

8. Tempany CM and McNeil BJ: Advances in biomedical imaging. JAMA 285: 562-567, 2001

9. Briganti A, Blute ML, Eastham JH, Graefen M, Heidenreich A, Karnes JR, Montorsi F and Studer UE: Pelvic lymph node dissection in prostate cancer. Eur Urol 55: 1251-1265, 2009.

10. Briganti A, Chun FK, Salonia A, Gallina A, Zanni G, Scattoni V Valiquette L, Rigatti P, Montorsi F and Karakiewicz PI: Critical assessment of ideal nodal yield at pelvic lymphadenectomy to accurately diagnose prostate cancer nodal metastasis in patients undergoing radical retropubic prostatectomy. Urology 69: 147-151, 2007.

11. Heidenreich A, Varga Z and Von Knobloch R: Extended pelvic lymphadenectomy in patients undergoing radical prostatectomy: High incidence of lymph node metastasis. J Urol 167: 1681-1686, 2002.

12. Clark T, Parekh DJ, Cookson MS, Chang SS, Smith ER Jr, Wells $\mathrm{N}$ and Smith J Jr: Randomized prospective evaluation of extended versus limited lymph node dissection in patients with clinically localized prostate cancer. J Urol 169: 145-148, 2003.

13. Bader P, Burkhard FC, Markwalder R and Studer UE: Is a limited lymph node dissection an adequate staging procedure for prostate cancer? J Urol 168: 514-518, 2002.
14. Burkhard FC and Studer UE: The role of lymphadenectomy in prostate cancer. Urol Oncol 22: 198-204, 2004.

15. Joslyn SA and Konety BR: Impact of extent of lymphadenectomy on survival after radical prostatectomy for prostate cancer. Urology 68: 121-125, 2006.

16. Bader P, Burkhard FC, Markwalder R and Studer UE: Disease progression and survival of patients with positive lymph nodes after radical prostatectomy. Is there a chance of cure? J Urol 169: 849-854, 2003

17. Daneshmand S, Quek ML, Stein JP, Lieskovsky G, Cai J, Pinski J, Skinner EC and Skinner DG: Prognosis of patients with lymph node positive prostate cancer following radical prostatectomy: long-term results. J Urol 172: 2252-2255, 2004.

18. Wagner M, Sokoloff M and Daneshmand S: The role of pelvic lymphadenectomy for prostate cancer-therapeutic? J Urol 179 408-413, 2008

19. Mitsuzuka K, Koie T, Narita S, Kaiho Y, Yoneyama T, Kawamura S, Tochigi T, Ohyama C, Habuchi T and Arai Y: Is pelvic lymph node dissection required at radical prostatectomy fo low-risk prostate cancer? Int J Urol 20: 1092-1096, 2013.

20. Perry-Keene J, Ferguson P, Samaratunga H, Nacey JN and Delahunt B: Total submission of pelvic lymphadenectomy tissues removed during radical prostatectomy for prostate cancer increases lymph node yield and detection of micrometastases. Histopathology 64: 399-404, 2014. 